\title{
Secondary Eclipse Photometry of the Exoplanet Wasp-5b With Warm Spitzer
}

\section{Citation}

Baskin, Nathaniel J., Heather A. Knutson, Adam Burrows, Jonathan J. Fortney, Nikole K. Lewis, Eric Agol, David Charbonneau, et al. 2013. Secondary Eclipse Photometry of the Exoplanet Wasp-5b With Warm Spitzer. The Astrophysical Journal 773, no. 2: 124. doi:10.1088/0004-637x/773/2/124.

\section{Published Version}

doi:10.1088/0004-637X/773/2/124

\section{Permanent link}

http://nrs.harvard.edu/urn-3:HUL.InstRepos:29990214

\section{Terms of Use}

This article was downloaded from Harvard University's DASH repository, and is made available under the terms and conditions applicable to Other Posted Material, as set forth at http:// nrs.harvard.edu/urn-3:HUL.InstRepos:dash.current.terms-of-use\#LAA

\section{Share Your Story}

The Harvard community has made this article openly available.

Please share how this access benefits you. Submit a story.

\section{Accessibility}




\title{
SECONDARY ECLIPSE PHOTOMETRY OF THE EXOPLANET WASP-5b WITH WARM SPITZER
}

\author{
Nathaniel J. Baskin ${ }^{1}$, Heather A. Knutson ${ }^{1}$, Adam Burrows ${ }^{2}$, Jonathan J. Fortney ${ }^{3}$, Nikole K. Lewis ${ }^{4}, 11$, Eric Agol ${ }^{5}$, \\ David Charbonneau ${ }^{6}$, Nicolas B. Cowan ${ }^{7}$, Drake Deming ${ }^{8}$, Jean-Michel Desert $^{1}$, Jonathan Langton $^{9}$, \\ Gregory Laughlin ${ }^{3}$, AND Adam P. Showman ${ }^{10}$ \\ ${ }^{1}$ Division of Geological and Planetary Sciences, California Institute of Technology, Pasadena, CA 91125, USA \\ ${ }^{2}$ Department of Astrophysical Sciences, Princeton University, Princeton, NJ 05844, USA \\ ${ }^{3}$ Department of Astronomy and Astrophysics, University of California, Santa Cruz, CA 95064, USA \\ ${ }^{4}$ Department of Earth, Atmospheric and Planetary Sciences, Massachusetts Institute of Technology, Cambridge, MA 02139, USA \\ ${ }^{5}$ Department of Astronomy, University of Washington, Box 351580, Seattle, WA 98195, USA \\ ${ }^{6}$ Harvard-Smithsonian Center for Astrophysics, Cambridge, MA 02138, USA \\ ${ }^{7}$ Center for Interdisciplinary Exploration and Research in Astrophysics, Northwestern University, Evanston, IL 60208, USA \\ ${ }^{8}$ Department of Astronomy, University of Maryland, College Park, MD 20742, USA \\ ${ }^{9}$ Department of Physics, Principia College, Elsah, IL 62028, USA \\ ${ }^{10}$ Lunar and Planetary Laboratory, University of Arizona, Tucson, AZ 85721, USA \\ Received 2013 March 14; accepted 2013 July 3; published 2013 August 1
}

\begin{abstract}
We present secondary eclipse photometry of the extrasolar planet WASP-5b taken in the 3.6 and $4.5 \mu \mathrm{m}$ bands with the Spitzer Space Telescope's Infrared Array Camera as part of the extended warm mission. By estimating the depth of the secondary eclipse in these two bands we can place constraints on the planet's atmospheric pressure-temperature profile and chemistry. We measure secondary eclipse depths of $0.197 \% \pm 0.028 \%$ and $0.237 \% \pm 0.024 \%$ in the $3.6 \mu \mathrm{m}$ and $4.5 \mu \mathrm{m}$ bands, respectively. For the case of a solar-composition atmosphere and chemistry in local thermal equilibrium, our observations are best matched by models showing a hot dayside and, depending on our choice of model, a weak thermal inversion or no inversion at all. We measure a mean offset from the predicted center of eclipse of $3.7 \pm 1.8$ minutes, corresponding to $e \cos \omega=0.0025 \pm 0.0012$ and consistent with a circular orbit. We conclude that the planet's orbit is unlikely to have been perturbed by interactions with another body in the system as claimed by Fukui et al.
\end{abstract}

Key words: eclipses - planetary systems - techniques: photometric

Online-only material: color figures

\section{INTRODUCTION}

Hot Jupiters are a class of extrasolar planet that, as the name suggests, are similar in size and composition to Jupiter but orbit very close to their parent star and have correspondingly high effective temperatures, ranging from $1000 \mathrm{~K}$ to over $3000 \mathrm{~K}$. By measuring the wavelength-dependent decrease in light during the secondary eclipse (when the planet passes behind its star), we can characterize the planet's emission spectrum and deduce its atmospheric properties (Deming et al. 2005; Charbonneau et al. 2005). These atmospheric properties include: whether or not the atmosphere has a temperature inversion and how well heat is redistributed from the planet's dayside to its nightside. In addition, the relative timing of transits and secondary eclipses can constrain the eccentricity of the planet's orbit.

To date Spitzer has measured secondary eclipses for nearly fifty extrasolar planets. The resulting studies indicate that hot Jupiters can be differentiated by the presence or absence of a strong thermal inversion in the planet's upper atmosphere (Burrows et al. 2008; Fortney et al. 2008; Barman 2008; Madhusudhan \& Seager 2009). Although it exhausted the last of its cryogen in 2009, the Spitzer Space Telescope's Infrared Array Camera remains functional in its 3.6 and $4.5 \mu \mathrm{m}$ bands (Fazio et al. 2004), and the telescope has continued to survey the emission spectra of hot Jupiters as part of Spitzer's extended warm mission.

Some groups have suggested that absorbers such as $\mathrm{TiO}$ in the upper atmosphere of hot Jupiters are responsible for atmospheric

\footnotetext{
11 Sagan Fellow
}

inversions (Hubeny et al. 2003; Fortney et al. 2008). However, large-scale atmospheric mixing would be required to preserve gaseous $\mathrm{TiO}$ in the upper atmosphere, as this molecule should condense in the deep interiors of most hot Jupiters. It is uncertain whether such macroscopic mixing should take place in a stably stratified atmosphere (Showman et al. 2009; Spiegel et al. 2009; Parmentier et al. 2013). In addition, temperature inversions have been observed on planets like XO-1b (Machalek et al. 2008) that have dayside temperatures below the condensation point of TiO. Sulfur-containing compounds have been proposed as an alternative absorber (Zahnle et al. 2009).

In this paper, we present observations of the transiting hot Jupiter WASP-5b. This planet is very dense compared to other planets in its class, suggesting the presence of a large, metalrich core (Anderson et al. 2008; Southworth et al. 2009; Madhusudhan \& Seager 2009; Gillon et al. 2009; Dragomir et al. 2011; Fukui et al. 2011; Pont et al. 2011; Hoyer et al. 2012). Knutson et al. 2010 reported evidence for a correlation between stellar activity and hot Jupiter emission spectra, where hot Jupiters with strong temperature inversions tend to orbit more quiet stars and more active stars typically host planets without inversions. This may be due to the fact that the more intense UV radiation from active stars destroys the compounds that are responsible for creating thermal inversions. WASP-5 is a modestly active G4V star with $\log \left(R^{\prime}\right)=-4.75$ and $S_{\mathrm{HK}}=0.215$, so it is not expected that this planet would have a strong temperature inversion. In addition, papers such as Cowan \& Agol (2011b), Showman \& Guillot (2002), and Perna et al. (2012) find that planets hotter than approximately $2000 \mathrm{~K}$ have a weak transport of energy to the nightside. With a predicted 


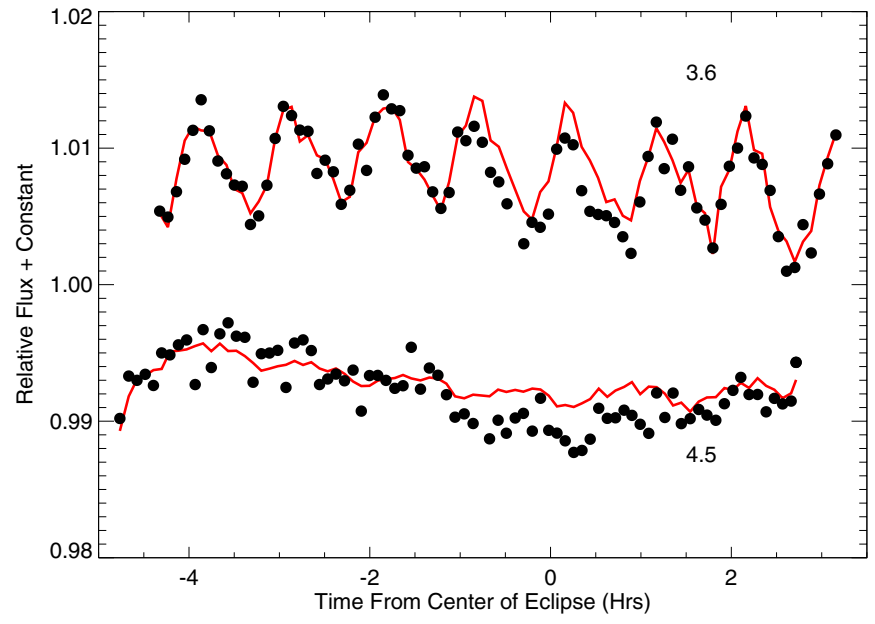

Figure 1. Raw photometry at 3.6 and $4.5 \mu \mathrm{m}$ vs. time from the center of the predicted secondary eclipse. The decorrelation functions to correct for intrapixel sensitivity are overplotted in red. Data are binned in sets of 25 points.

(A color version of this figure is available in the online journal.)

equilibrium temperature of $1720 \mathrm{~K}$ for the case of zero albedo and full day-night redistribution of energy, WASP-5b is near the boundary of this transition. In this paper, we will test the correlations proposed by Knutson et al. (2010) and Cowan \& Agol (2011b) by constraining the atmospheric properties of WASP-5b.

In a previous study, Fukui et al. (2011) found that the intervals between WASP-5b's transits do not appear to be constant, which could indicate the presence of an additional body in the system perturbing the planet's orbit. Hoyer et al. (2012), however, disputes the claims of such transit timing variations (TTVs). In Section 3.2, we present the first measurements of this planet's secondary eclipse times and discuss the implications of our measurements for the proposed perturber.

\section{OBSERVATIONS AND METHODS}

We began by extracting photometry from calibrated images in the $3.6 \mu \mathrm{m}$ and $4.5 \mu \mathrm{m}$ bands from the Spitzer Space Telescope following the methods described in Knutson et al. (2012) and Lewis et al. (2013). Each channel consists of $211512 \mathrm{~s}$ images acquired over a period of $7.7 \mathrm{hr}$. The basic calibrated data files used were dark-subtracted, linearized, flat-fielded, and fluxcalibrated. We correct for transient "hot pixels" in a $20 \times 20$ pixel box around the star by removing intensity values $>3 \sigma$ from the median value at that pixel position in the surrounding 50 images. These values are then replaced by the median. In addition, to further reduce the noise in the data we trimmed the first 53 minutes from channel $1(3.6 \mu \mathrm{m})$ data and the first 15 minutes from channel $2(3.6 \mu \mathrm{m})$ data, which exhibit larger deviations in position. As a test we repeated our fits with no trimming and found that our best-fit eclipse depths change by less than $1 \sigma$.

We performed aperture photometry using the aper routine from the IDL Astronomy Library (http://idlastro.gsfc.nasa.gov/ homepage.html) with radii ranging from 2.0 to 3.0 pixels in intervals of 0.1 pixels, and from 3.0 to 5.0 pixels in half-pixel intervals. We found that setting the aperture for channel 2 equal to 2.2 pixels produced the smallest root mean square (rms) scatter in the data. We obtained superior results in channel 1 using a time-varying aperture proportional to the square root of the noise pixel value (Knutson et al. 2012; Lewis et al. 2013) minus 0.1 pixels. In channel 2 , however, using a time varying

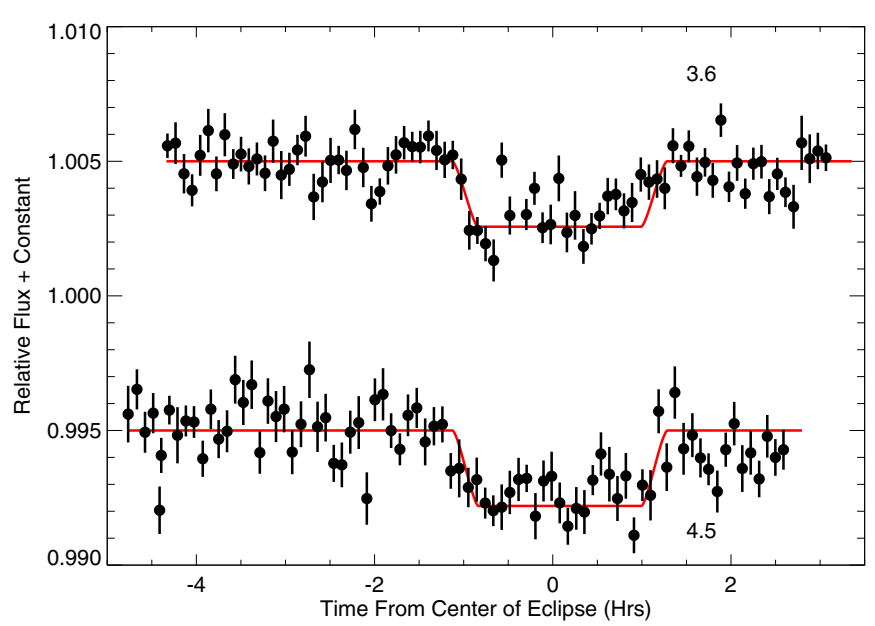

Figure 2. Photometry and best-fit eclipse curve for both wavebands after decorrelation versus time from the predicted center of the secondary eclipse. The data are binned in sets of 25 points, with errors calculated as the standard deviation of the points in each bin divided by the square root of the number of points.

(A color version of this figure is available in the online journal.)

aperture increased the scatter, so a fixed size aperture was selected. Our median aperture size for channel 1 was 1.7 pixels. We determined the position of the star in both channels using flux-weighted centroiding with a radius of 5.0 pixels.

We next correct for the dominant instrument effect in the two bands. Since the sensitivity of individual pixels varies from the center out to the edge, the apparent flux of the star will fluctuate with the movement of the telescope. To correct for this intrapixel sensitivity variation, we fit the data with quadratic functions in the $x$ and $y$ positions. For channel 1, the measured flux $f$ is given by

$f=f_{0}\left(c_{1}\left(x-x_{0}\right)^{2}+c_{2}\left(y-y_{0}\right)^{2}+c_{3}\left(x-x_{0}\right)+c_{4}\left(y-y_{0}\right)+c_{5}\right)$

where $f_{0}$ is the incident flux, $x$ and $y$ are the positions of the star on the array, $x_{0}$ and $y_{0}$ are the median positions over the time series, and $c_{1-5}$ are free parameters. We also tried fitting cubic and linear functions, as well as adding an $x y$ term, but found that a quadratic function yielded the lowest value of the Bayesian Information Criterion in both channels. Figure 1 shows the raw data together with our best-fit quadratic functions. Figure 2 displays the normalized data with best-fit eclipse models for each channel.

We determined our best-fit parameters from a $\chi^{2}$ minimization and used a Markov Chain Monte Carlo (MCMC) method (Ford 2005; Winn et al. 2007) with $10^{5}$ steps to determine the corresponding uncertainties in our fitted parameters. We set the uncertainty on individual points in our time series equal to the standard deviation of the best-fit residuals in each band $(0.37 \%$ and $0.43 \%$ in channels 1 and 2, respectively). We find that the rms of our best-fit residuals is a factor of 1.2 (channel 1) and 1.1 (channel 2) times higher than the photon noise limit. We used seven free parameters in each channel, including the eclipse depths, timing offsets, and the intrapixel sensitivity corrections. The planetary and stellar radii, orbital period, and orbital inclination were set to the values given in Hoyer et al. (2012). Once the chain is completed we find the point where the $\chi^{2}$ value for the chain first moves below the median $\chi^{2}$ value over the entire chain, and trim the steps up to that point. We define the uncertainty for each parameter as the symmetric range over 
Table 1

Best-fit Values for the Secondary Eclipse Depth and Time ${ }^{\mathrm{a}}$

\begin{tabular}{lcccc}
\hline \hline $\begin{array}{l}\text { Wavelength } \\
(\mu \mathrm{m})\end{array}$ & $\begin{array}{c}\text { Eclipse Depth } \\
(\%)\end{array}$ & $\begin{array}{c}\text { Brightness Temperature } \\
(\mathrm{K})\end{array}$ & $\begin{array}{c}\text { Center of Eclipse } \\
(\mathrm{BJD})\end{array}$ & $\begin{array}{c}\text { Eclipse Offset } \\
(\mathrm{min})\end{array}$ \\
\hline 3.6 & $0.197 \pm 0.028$ & $2210 \pm 140$ & $2455200.4279 \pm 0.0016$ & $4.0 \pm 2.4$ \\
4.5 & $0.237 \pm 0.024$ & $2130 \pm 110$ & $2455174.3726 \pm 0.0019$ & $3.3 \pm 2.8$ \\
\hline
\end{tabular}

Note. ${ }^{a}$ Both eclipse times are given in the BJD $_{\text {UTC }}$ time standard; to convert to BJD $_{\mathrm{TT}}$ simply add $66.184 \mathrm{~s}$. We calculate the timing offsets using the ephemeris from Fukui et al. (2011) and accounting for the 26.78 s light-travel time in this system (Loeb 2005). We also include the uncertainty in the predicted eclipse times at the epoch of our observations, but this is only $39 \mathrm{~s}$ and has a negligible effect on the combined uncertainty.

which the probability distribution contains $68 \%$ of the points around the median. The distributions for all parameters were approximately Gaussian, and the eclipse depths and times were not strongly correlated with any of the other fit parameters. Our probability distributions for the eclipse depths and times were smooth and well-sampled, indicating that the chains had fully converged. See Table 1 for the best-fit eclipse depths and times.

We also calculate error bars using the "prayer-bead" method Gillon et al. (2009), which involves shifting the residuals of the data in single point increments and recalculating the best-fit eclipse depth and time. For a comparison of this method versus other approaches for treating time-correlated noise, see Carter \&Winn (2009). We find that the prayer-bead method yields error bars consistent with those of the MCMC, and we chose to use the larger of the two errors in each case. For channel 1, we used the prayer-bead error for both the eclipse depth and center of eclipse time $(0.028 \%$ and $0.039 \mathrm{hr}$, respectively) as opposed to the MCMC error ( $0.027 \%$ and $0.038 \mathrm{hr})$. For channel 2, we used the MCMC for the eclipse depth $(0.024 \%$ as opposed to $0.022 \%$ from the prayer-bead) and both methods give errors of $0.046 \mathrm{hr}$ for the eclipse time.

\section{DISCUSSION}

\subsection{Atmospheric Temperature Structure}

We consider two classes of atmospheric models in this paper. The first, following the methods of Fortney et al. (2008) examines the impact of $\mathrm{TiO}$ as an absorber in the upper atmosphere. These models parameterize the redistribution of heat by adjusting the incident flux at the top of the atmosphere. In Figure 3, four models are shown. The red and blue models represent a planet with a hot dayside that redistributes little heat to the nightside. The green and purple models characterize an atmosphere in which heat is evenly distributed between the planet's day and night sides. The blue and purple models contain gas-phase $\mathrm{TiO}$, which acts as an absorber in the planet's upper atmosphere. The red and green models contain no TiO. We find that the slope between the band averaged flux ratios on the blue model is too steep to fit both of our observed values. As seen in the figure, our observations are best matched by the red model, which has no thermal inversion and poor redistribution of energy from the dayside to the nightside.

The second class of models follow the methods of Burrows et al. (2008). As opposed to modifying the atmospheric levels of $\mathrm{TiO}$, this method utilizes a nonspecific gray absorber at low pressures. A heat sink is added at depth to allow for the redistribution of heat from the planet's dayside to its nightside. We use the dimensionless parameter $P_{n}$ to specify the heat redistribution, where $P_{n}=0.5$ indicates evenly distributed energy among both the day and night sides of the planet, and $P_{n}=0.0$ indicates no heat redistribution. This model also puts an extra

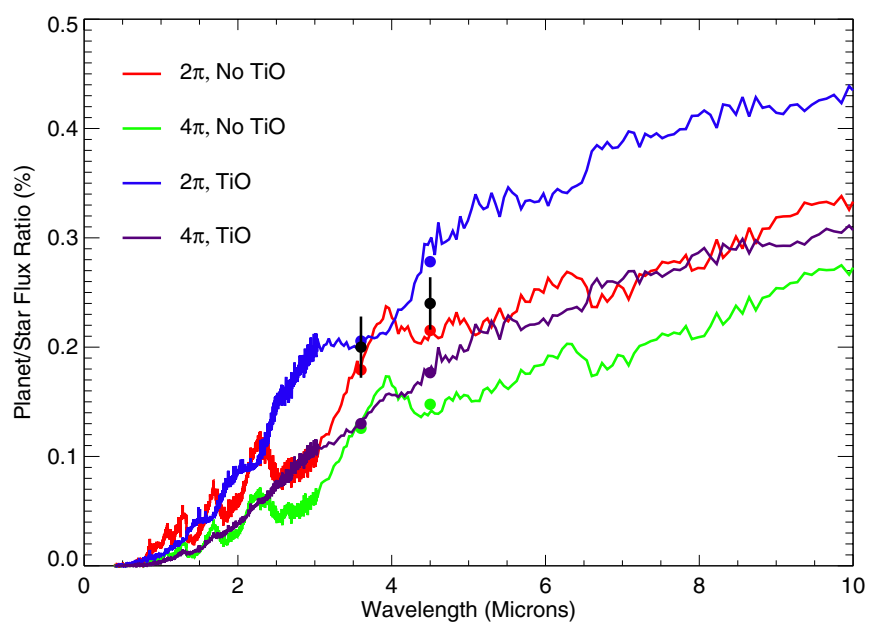

Figure 3. Dayside planet/star flux ratio vs. wavelength for four model atmospheres (Fortney et al. 2008) with the band-averaged flux ratios for each model superposed (colored circles). Stellar fluxes were calculated using a $5700 \mathrm{~K}$ PHOENIX stellar atmosphere model (Hauschildt et al. 1999). The observed contrast ratios are overplotted as the black circles, with uncertainties shown. Two models contain gas-phase $\mathrm{TiO}$, which produces a high-altitude temperature inversion (Parmentier et al. 2013). In the figure, the red and blue models represent a planet that radiates heat only from the dayside. The red and blue models represent a planet that radiates heat only from the dayside, while the green and purple models distribute heat equally between the two hemispheres.

(A color version of this figure is available in the online journal.)

absorber with an optical opacity, $\kappa$, in the upper atmosphere to heat it by partial absorption of the incident stellar flux in visible wavelengths. An enhancement in optical wavelength opacity in the planet's atmosphere will produce a thermal inversion, but importantly raises the temperature of the outer atmosphere to better reproduce the photometric signatures of a subset of measured hot Jupiters. The character of such an absorber, and whether it is a molecule or haze, are currently unknown. Figure 4 shows three of these models. Our observations are best matched by the green model, which indicates a weak temperature inversion and little heat distribution between the sides of the planet, in good agreement with the Fortney et al. (2008) models.

We calculate brightness temperatures for the planet in both bands using a PHOENIX model atmosphere for the star with stellar parameters from Doyle et al. (2012) and find planetary brightness temperatures of $2210 \pm 140 \mathrm{~K}$ at $3.6 \mu \mathrm{m}$ and $2090 \pm$ $120 \mathrm{~K}$ at $4.5 \mu \mathrm{m}$. If the planet has an albedo of zero and reradiates the absorbed flux uniformly over its entire surface, we calculate a predicted equilibrium temperature of $1700 \mathrm{~K}$. If it re-radiates this energy from the dayside hemisphere alone, this temperature increases to $2030 \mathrm{~K}$. The measured brightness temperatures therefore provide a model-independent indication that WASP-5b has a hot dayside and relatively inefficient day-night recirculation. We consider whether or not this is 


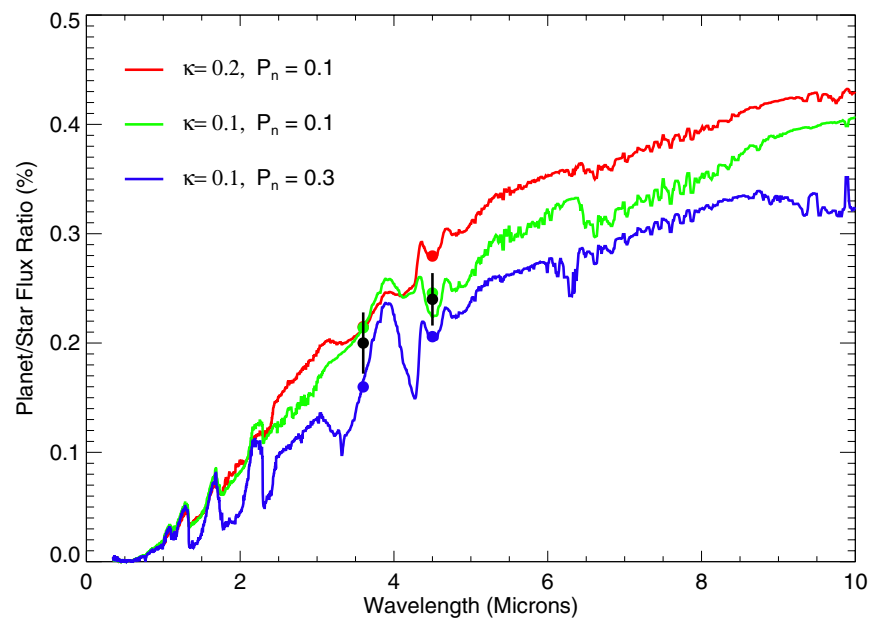

Figure 4. Dayside planet/star flux ratio vs. wavelength for three model atmospheres (Burrows et al. 2008) with the band-averaged flux ratios for each model superposed (colored circles). Stellar fluxes were calculated using a 5700 K ATLAS stellar atmosphere model (Kurucz 2005). The observed contrast ratios are overplotted as the black circles, with uncertainties shown. The model parameter $\kappa$ is related to the atmosphere's opacity, while $p$ is related to the heat redistribution between the day and night sides of the planet $\left(P_{n}=0.0\right.$ indicates no heat redistribution, and $P_{n}=0.5$ indicates complete redistribution).

(A color version of this figure is available in the online journal.)

typical of the class of hot Jupiters by plotting the measured surface fluxes for all planets with published secondary eclipses in the 3.6 and $4.5 \mu \mathrm{m}$ Spitzer bands and comparing these fluxes to the values for a blackbody at the predicted equilibrium temperature (see Figure 5). We find that these planets universally exhibit flux excesses relative to the coolest predicted blackbody (corresponding to efficient day-night circulation) in at least one, and sometimes both, Spitzer bands. We do not find any clear correlation between flux excesses and the predicted planet temperature, although planets orbiting active stars tend to lie toward the lower right region of the plot.

Our results are consistent with the correlation between stellar activity and hot Jupiter emission spectra, as described in Knutson et al. (2010). According to this study, hot Jupiters with strong temperature inversions tend to orbit more quiet stars, while more active stars typically host planets without inversions. WASP-5 is a G4V star with $\log \left(R^{\prime}\right)=-4.75$ and $S_{\mathrm{HK}}=0.215$, indicating that the star is modestly active. This is consistent with our results from the atmospheric models (Section 3.1), which suggest the presence of either a weak thermal inversion or none at all. In addition, the low heat redistribution of WASP-5b is consistent with the predictions of Cowan \& Agol (2011b).

\subsection{Orbital Eccentricity}

The timing of the secondary eclipse depends on the eccentricity of the planet's orbit. As a result, we can utilize the predicted and observed times of eclipse to constrain $e \cos \omega$, where $e$ is the orbital eccentricity and $\omega$ is the argument of pericenter (Charbonneau et al. 2005). We note that if the planet has a non-uniform dayside brightness distribution we may measure an apparent offset in the secondary eclipse time; following the model of Cowan \& Agol (2011a), as implemented in Agol et al. (2010), we calculate that the maximum expected time offset due to an offset hot spot would be $37 \mathrm{~s}$ or less than $1 \sigma$ for our measurement.

We calculate our predicted eclipse times using the ephemeris from Fukui et al. (2011), which has smaller errors than the

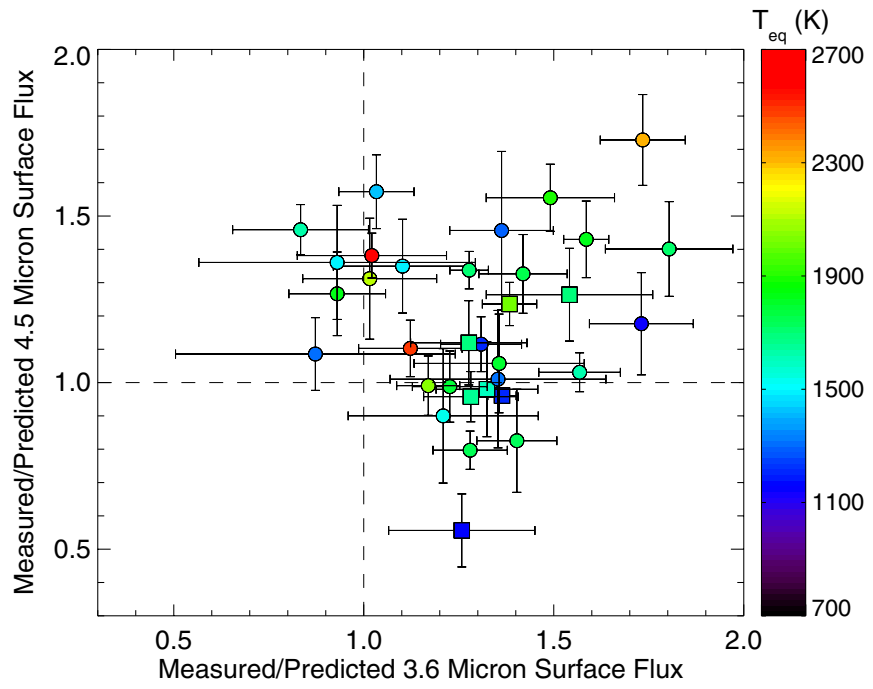

Figure 5. Ratio of measured to predicted planet surface flux in the $3.6 \mathrm{vs}$. $4.5 \mu \mathrm{m}$ Spitzer bands for all planets with published secondary eclipse detections at these two wavelengths. The colors of the points reflect the predicted equilibrium planet temperature $T_{\text {eq }}$, which is calculated assuming that the planet has an albedo of zero and re-radiates energy uniformly over its entire surface. Predicted fluxes are calculated assuming the planet radiates as a blackbody at this temperature. Measured fluxes are calculated using published secondary eclipse depths and assuming a PHOENIX atmosphere model for the star. For planets with eccentricities greater than 0.05 measured at $3 \sigma$ significance or higher we show the orbit-averaged temperature. Planets orbiting active stars (defined as having $\log \left(R_{\mathrm{HK}}^{\prime}\right)$ (Knutson et al. 2010) greater than -4.9 and a stellar effective temperature less than $6200 \mathrm{~K}$ ) are shown as filled squares, all other planets are plotted as filled circles.

(A color version of this figure is available in the online journal.)

ephemeris reported by Hoyer et al. (2012); this may be due to Fukui et al.'s inclusion of both the radial velocity and transit data in their fits. Using these values and accounting for the $27 \mathrm{~s}$ light travel time we find a mean offset from the predicted center of eclipse of $3.7 \pm 1.8$ minutes, translating to an $e \cos \omega$ value of $0.0025 \pm 0.0012$. This indicates that, unless our line of sight is aligned with the planet's semi-major axis, the orbit is circular. Our result is also consistent with the $95 \%$ confidence upper limit of 0.026 on the planet's eccentricity from Husnoo et al. (2012). We note that Fukui et al. (2011) claimed to detect TTVs for this planet, although this claim has been disputed by Hoyer et al. (2012). If the planet is being perturbed by an additional body in the system we would expect these perturbations to excite its orbital eccentricity and to maintain that eccentricity despite ongoing circularization from tidal interactions with the parent star. The lack of any orbital eccentricity therefore provides further evidence against the presence of a perturbing body in this system.

\section{CONCLUSIONS}

We observed secondary eclipses of the extrasolar planet WASP-5b in the 3.6 and $4.5 \mu \mathrm{m}$ bands, and compared our measurements with atmospheric models generated according to the methodology of Burrows et al. (2008) and Fortney et al. (2008). Our eclipse values of $0.197 \% \pm 0.028 \%$ and $0.237 \% \pm 0.024 \%$ in these bands are best matched by models indicating a weak thermal inversion or no inversion at all. Our measurements are consistent with the observed correlation between stellar activity and the presence of thermal inversions presented in Knutson et al. (2010). In addition, these one-dimensional atmospheric models all indicate minimal day-night redistribution of energy 
for WASP-5b, with the Burrows models suggesting a redistribution factor of 0.1 , consistent with the prediction of Cowan \& Agol (2011b). Measurements at additional wavelengths would be helpful to confirm or reject the presence of the inversion. These results further demonstrate the utility of warm Spitzer in characterizing the properties of hot Jupiter atmospheres, despite being limited to the 3.6 and $4.5 \mu \mathrm{m}$ channels. Additional measurements in the $J, H$, and $K$ bands would also allow us to constrain the metallicity of WASP-5b's atmosphere, which is likely to be enhanced relative to other hot Jupiters.

By measuring the mean timing offset from the predicted center of eclipse ( $3.7 \pm 1.8$ minutes $)$, we calculate the parameter $e \cos \omega$ to be $0.0025 \pm 0.0012$. This indicates that the planet's orbit must be circular unless our line of sight is well-aligned with the semi-major axis. These observations, along with those in Hoyer et al. (2012), argue against the possibility of ongoing perturbations from an additional body in the WASP-5 system.

\section{REFERENCES}

Agol, E., Cowan, N. B., Knutson, H. A., et al. 2010, ApJ, 721, 1861 Anderson, D. R., Gillon, M., Hellier, C., et al. 2008, MNRAS, 387, L4 Barman, T. S. 2008, ApJL, 676, L61

Burrows, A., Budaj, J., \& Hubeney, I. 2008, ApJ, 678, 1463

Carter, J. A., \& Winn, J. N. 2009, ApJ, 704, 51

Charbonneau, D., Allen, L. E., Megeath, S. T., et al. 2005, ApJ, 626, 523

Cowan, N. B., \& Agol, E. 2011a, ApJ, 726, 82

Cowan, N. B., \& Agol, E. 2011b, ApJ, 729, 54
Deming, D., Seager, S., Richardson, L. J., \& Harrington, J. 2005, Natur, 434, 740

Doyle, A. P., Smalley, B., Maxted, P. F. L., et al. 2012, MNRAS, 428, 3164

Dragomir, D., Kane, S. R., Pilyavsky, G., et al. 2011, AJ, 142, 115

Fazio, G. G., Hora, J. L., Allen, L. E., et al. 2004, ApJS, 154, 10

Ford, E. B. 2005, AJ, 129, 1706

Fortney, J. J., Lodders, K., Marley, M. S., \& Freedman, R. S. 2008, ApJ, 678 1419

Fukui, A., Narita, N., Tristram, P. J., et al. 2011, PASJ, 63, 287

Gillon, M., Smalley, B., Hebb, L., et al. 2009, A\&A, 496, 259

Hauschildt, P., Allard, F., Ferguson, J., Baron, E., \& Alexander, D. R. 1999, ApJ, 525,871

Hoyer, S., Rojo, P., \& López-Morales, M. 2012, ApJ, 748, 22

Hubeny, I., Burrows, A., \& Sudarsky, D. 2003, ApJ, 594, 1011

Husnoo, N., Pont, F., Mazeh, T., et al. 2012, MNRAS, 422, 3151

Knutson, H. A., Howard, A. W., \& Isaacson, H. 2010, ApJ, 720, 1569

Knutson, H. A., Lewis, N., Fortney, J. J., et al. 2012, ApJ, 754, 22

Kurucz, R. 2005, MSAIS, 8, 14

Lewis, N. K., Knutson, H. A., Showman, A. P., et al. 2013, ApJ, 766, 95

Loeb, A. 2005, ApJL, 623, L45

Machalek, P., McCullough, P. R., Burke, C. J., et al. 2008, ApJ, 684, 1427

Madhusudhan, N., \& Seager, S. 2009, ApJ, 707, 24

Parmentier, V., Showman, A. P., \& Lian, Y. 2013, A\&A, submitted (arXiv: 1301.4522)

Perna, R., Heng, K., \& Pont, F. 2012, ApJ, 751, 59

Pont, F., Husnoo, N., Mazeh, T., \& Fabrycky, D. 2011, MNRAS, 414, 1278

Showman, A. P., Fortney, J. J., Lian, Y., et al. 2009, ApJ, 699, 564

Showman, A. P., \& Guillot, T. 2002, A\&A, 385, 166

Southworth, J., Hinse, T. C., Burgdorf, M. J., et al. 2009, MNRAS, 399, 287

Spiegel, D. S., Silverio, K., \& Burrows, A. 2009, ApJ, 699, 1487

Winn, J. N., Holman, M. J., \& Fuentes, C. I. 2007, AJ, 133, 11

Zahnle, K., Marley, M. S., Freedman, R. S., Lodders, K., \& Fortney, J. J. 2009, ApJL, 701, L20 